\title{
Creación y funcionamiento de la comisión de formación continuada de las profesiones sanitarias y de su sistema acreditador
}

\author{
The Spanish Commission of Continuing Education of the National Health System
}

\author{
Miguel Javier Rodríguez Gómez* y Luis Pallarés Neila** \\ * Subdirector General de Ordenación Profesional. Ministerio de Sanidad y Consumo \\ ** Director de Programas. Ministerio de Sanidad y Consumo
}

En el contexto de la introducción de la Formación Médica Continuada (FMC) sistematizada, que viene ocurriendo en los países occidentales en las últimas décadas, la implementación de un sistema de acreditación ha supuesto un avance considerable. Tal sistema, auspiciado por la Comisión de Formación Continuada del Sistema Nacional de Salud, en 1977, en España, ha supuesto un hito a nivel europeo, dado que únicamente Italia dispone de dicho sistema acreditador. El sistema español de acreditación se basa en la valoración de los Componentes Cualitativo y Cuantitativo de la actividad. El primero se valora mediante una escala cualitativa de Licker, por parte de evaluadores externos y el segundo tiene en cuenta el número de horas lectivas, convenientemente ponderado.

En la actualidad, teniendo en cuenta la existencia de un marco legislativo adecuado, el futuro del sistema español de acreditación presenta unos indudables aspectos positivos, que permiten contemplar el futuro con optimismo.

Palabras clave: Formación médica continuada, acreditación de la formación médica continuada, créditos de formación médica continuada.
In the context of the Continuing Medical Education (CME) systems that are being introduced in Western countries in the last decades, the implementation of a CME accreditation system is a very important step. Such a system, promoted by the Spanish Commission of Continuing Education of the National Health System in 1997, represents a crucial feature at the European level, since only Italy and Spain have the aforementioned accreditation system.

The Spanish CME accreditation system is mainly based on the assessment of two core elements: the Qualitative Factor and the Quantitative Factor. The former is assessed through a qualitative Licker scale applied by external assessors. The Quantitative Factor relates to the learning hours, adequately weighted.

Currently, taking into account the existence of an adequate regulatory framework, the future of the Spanish CME accreditation system can be optimistically viewed, mainly based on its positive aspects.

Key words: Continuing medical education, continuing medical education accreditation, continuing medical education credits

La formación es un elemento esencial para el desempeño correcto y eficaz de un ejercicio profesional de calidad; sin ella difícilmente se pueden plantear objetivos profesionales para resolver un problema o satisfacer una necesidad. De la misma manera, para el desarrollo de la proposición de salud y la práctica 
de la educación para la salud, es imprescindible, incluso un condicionante, disponer de una formación suficiente y de calidad, coherente con las funciones y el papel del profesional médico ${ }^{1}$.

El momento y las circunstancias en las que se adquiere la formación vienen a determinar los currículos formativos, así como los centros e instituciones responsables de su desarrollo. De esta manera podemos diferenciar tres niveles formativos (Tabla I) ${ }^{2}$ :

\section{Tabla 1. NIVELES FORMATIVOS}

\section{- EDUCACIÓN SANITARIA FORMAL - Pregrado$$
\text { - Postgrado }
$$$$
\text { - EDUCACIÓN SANITARIA CONTINUADA }
$$

1. La formación inicial o pregrado, competencia de la Universidad, es aquella que se establece para la obtención del título y legitima para el ejercicio profesional. En este caso, los currículos universitarios e itinerarios formativos se han de corresponder con el desarrollo del papel profesional ulterior.

2. La formación de postgrado es de carácter académico y orientada a la consecución de una titulación y es competencia de la Universidad. Se corresponde con los estudios posteriores a la Licenciatura y su finalidad es adquirir un cierto grado de especialización.

3. La formación continuada es aquella formación no reglada que sigue el profesional después de finalizar su formación básica y/o especializada. No tiene como finalidad la obtención de un título o diploma, sino mantener o aumentar su competencia profesional. Al ser una enseñanza libre, las actividades de formación continuada recaen tanto en instituciones o centros de carácter público como privados.

Los primeros programas de formación médica continuada aparecieron en 1911 en la Facultad de Medicina de la Universidad de Kansas (EE.UU.). En la actualidad, son muchos los países en los que funcionan sistemas de formación médica continuada, de carácter muy diferente según el distinto protagonismo de los organismos de la administración sanitaria o de las propias corporaciones profesionales y de acuerdo con la obligatoriedad o no de la evaluación.

Desde un punto de vista conceptual, en 1973, la Organización Mundial de la Salud definió la formación médica continuada como la formación que un médico recibe después de finalizar su instrucción básica o, en su caso, después de terminar cualquier estudio adicional, para ejercer como médico general o especialista. Se trata de una formación encamina- da a mejorar su competencia profesional sin el objetivo de conseguir un nuevo título o diploma.

No existe una única definición del concepto de formación médica continuada. Cada definición aporta nuevas formas de entender este concepto.

Gallois la define como una garantía de la prestación y de la adaptación del médico a la evolución permanente del sistema sanitario, y como instrumento fundamental de regulación del mismo.

El Accreditation Council for Continuing Medical Education de EE.UU. añade que su contenido es el conjunto de conocimientos y habilidades basado en las ciencias médicas básicas, la medicina clínica y la provisión de asistencia sanitaria a la población. Este conjunto de conocimientos y habilidades debe ser pertinente, es decir, debe responder a las necesidades de salud y ser congruente con los recursos de la comunidad y los planes para mejorarla.

Otros autores definen la formación médica continuada como un conjunto de alternativas educativas centradas en el desarrollo profesional, ya sea a través de cursos y actividades de carácter complementario y sistematizado, o de publicaciones y difusión de temas específicos de un determinado campo del conocimiento.

Esta formación se ha realizado siempre de una $u$ otra forma, pues desde antiguo el médico ha empleado parte de su tiempo en esta tarea de aprendizaje constante. Su actividad se ajusta a una preocupación creciente por el control social que ha motivado que el profesional médico deba acreditar objetivamente ante la sociedad el mantenimiento de su competencia. La comunidad médica europea recomienda la práctica habitual de la formación médica continuada. Así, existe a nivel del Parlamento Europeo una propuesta sobre la obligatoriedad de incorporar la formación médica continuada como garantía del ejercicio profesional médico.

Este requerimiento de una formación continuada no es improcedente ni arbitrario, ya que el actual ritmo de recambio o innovaciones en el saber y en las competencias propias, hace que la caducidad del conocimiento sea muy alta. Se sabe que a los pocos años de haber finalizado, un alto porcentaje de la formación, de las competencias aprendidas e, incluso de las destrezas desarrolladas como producto de $\mathrm{su}$ ejercicio y de las experiencias vividas, queda obsoleto y debe ser reemplazado por un nuevo conocimiento y renovación del aprendizaje. De modo que la evaluación cíclica o periódica, en lapsos que pueden diferir según cual sea la dedicación o la especialidad que se ejerce, es razonable como garantía de la idoneidad profesional. 
Tabla 2.

LA NECESIDAD DE UN SISTEMA DE FORMACIÓN CONTINUADA REQUIERE UN SISTEMA DE ACREDITACIÓN

Esta situación de necesidad de disponer de un sistema de formación continuada requiere adherirla a algún medio de acreditación solvente y funcional. Para ello hay modelos tanto de larga como de reciente tradición que pueden ser apreciados como experiencias contrastables (Tabla II).

En los Estados Unidos de Norteamérica, con su estructura de libre competencia y con tradición acreditadota de las sociedades científicas, el sistema acreditador de la formación médica continuada es administrado por la ACCME. Este es un organismo conformado por diversas asociaciones (la Association of American Medical Collage, la Association of Hospital Medical Education, el American Borrad of Medical Specialties, el Council of Medical Specialties Societies, la American Medical Association, ...), más un representante del Gobierno Federal y otro del Estado. Sus funciones se refieren a acreditar las entidades nacionales que imparten formación médica continuada, a reconocer las entidades acreditadotas de los estados y a controlar todo el sistema.

En Europa, la formación médica continuada, en cuanto a las actividades propiamente formativas, tiende a ser similar, habiéndose producido un notable desarrollo de proveedores públicos y privados que ofrecen oportunidades de formación a los médicos de los distintos países. En lo que respecta a la acreditación, ningún país salvo España, ha implementado aún un sistema nacional de acreditación, a excepción de los que desde hace años existen a cargo de Sociedades Científicas, Universidades o Colegios de Médicos o equivalentes. Los países de la Europa del Este suponen una excepción, pues ya contaban con programas de formación médica continuada obligatorios y periódicos.

Aunque muchos países están planteándose desarrollar sistemas de acreditación que a la larga puedan servir para toda la comunidad europea, el gran reto consiste en evitar modelos que pequen de excesiva burocratización lo que les llevaría a ser inoperantes. Además, la internalización del conocimiento y la pérdida de referencias concretas hacen, ahora, más importante que nunca la evaluación de la calidad y la construcción de sistemas de acreditación de ésta.

En España, el informe de la Comisión de Análisis y Evaluación del Sistema Nacional de Salud,
"Informe Abril", señala que la formación continuada del personal sanitario debe ser fundamento permanente del sistema. Añade que esta formación continuada supone la mejor garantía de cualificación profesional, incentivo en su trabajo diario, motivación personal y la seguridad para la sociedad de que sus actuaciones llevan implícita una valoración equilibrada entre el beneficio social colectivo y el uso de los recursos en su justa medida. Una de las recomendaciones de esta Comisión señala que: "Deberían impulsarse los procesos de formación continuada y la actualización de las competencias profesionales".

En el mismo año que el "Informe Abril" (1991), el Comité de Prioridades del Sistema de Salud del Gobierno de Holanda señala que los profesionales sanitarios deben ser responsables de la calidad de sus actos. Para ello da un papel preferente a las asociaciones científicas y organizaciones profesionales a la hora de establecer protocolos, principios generales y listas esenciales para obtener unos cuidados de salud apropiados. El informe indica que debe prestarse una especial atención a la educación de los profesionales sanitarios en aspectos económicos, éticos, legales y psicológicos del Sistema de Salud.

Esta preocupación social para que los profesionales sanitarios sean competentes para realizar su trabajo, ha dado lugar a iniciativas para introducir en nuestro país medidas para favorecer la participación del profesional en programas de formación continuada.

La Ley 14/1986, de 25 de abril, General de Sanidad, en el apartado 14 de su artículo 18, otorga a las Administraciones Públicas, a través de sus Servicios de Salud y sus órganos competentes en cada caso, la mejora y adecuación de las necesidades de la formación del personal al servicio de la organización sanitaria. Para ello y de acuerdo con el artículo 104 de la Ley, se dispondrá de toda la estructura asistencial del sistema sanitario para ser utilizada para la docencia pregraduada y continuada de los profesionales.

Por otro lado, los Estatutos de Autonomía, los Decretos de transferencia de competencias, funciones y servicios a las Comunidades Autónomas y los Decretos sobre Organización y Competencias de los Órganos de los Departamentos de Salud de las diferentes Comunidades Autónomas, otorgan a las mismas competencias en la materia; concretamente, sobre la implantación y desarrollo de programas de formación continuada del personal sanitario.

Es de destacar que el Consejo Interterritorial del Sistema Nacional de Salud aprobó, en su reunión del día 5 de octubre de 1988 , un documento sobre 
"Criterios generales de coordinación sanitaria: Conjunto mínimo de objetivos e indicadores sanitarios".

Este documento fue ratificado en la reunión del Consejo del día 19 de julio de 1989, señalando como objetivo general, en su apartado 5 "la garantía de calidad de los servicios prestados por el sistema sanitario". Este objetivo general contiene tres objetivos específicos que están relacionados con la formación continuada:

- Garantizar la calidad de los servicios sanitarios, estableciendo programas homologados de garantía de calidad total en los diferentes centros sanitarios.

- Procurar la existencia de una carrera profesional para el personal al servicio del Sistema Nacional de Salud. En esta línea, crear programas de formación continuada y un sistema de incentivos que discrimine según rendimiento, mérito y calidad.

- Desarrollar programas de control y mejora de calidad asistencial en centros y servicios sanitarios, y proporcionar sistemas de acreditación que refuercen el interés de los profesionales, servicios y centros en la mejora del proceso asistencial y en la actualización profesional, científica e investigadora.

Tanto a nivel nacional como internacional se ha venido legislando en esta materia.

Como respuesta a todas estas recomendaciones y a las diversas demandas profesionales, y con el fin de paliar esta situación, se firmó el Convenio de Conferencia Sectorial ${ }^{3}$ que condujo a que en el seno del Consejo Interterritorial se creara, en 1997, la Comisión de Formación Continuada del Sistema Nacional de Salud, cuyo funcionamiento y resultados han sido claves en todo el proceso de acreditación porque, gracias a su trabajo y dedicación, hoy podemos decir que la evaluación de la formación continuada es una realidad en funcionamiento. Para que ello haya sido posible debemos agradecer la colaboración y buena disposición de todas las administraciones e instituciones del sector sanitario, que han mostrado su apoyo a estas iniciativas y han asumido las responsabilidades que por su representatividad debían afrontar en esta materia y sin cuya colaboración no podríamos hablar ahora de realidades en una faceta tan sentida y necesaria para profesionales y ciudadanos que requieren, cada día con más intensidad, nuevos y mejores servicios asistenciales y, por lo tanto, una mejor preparación y formación de los que los llevan a cabo.

La Comisión de Formación Continuada del Sistema Nacional de Salud celebró su sesión consti- tutiva el 22 de enero de 1998 en Sevilla, donde se acuerda la creación de un Grupo de Trabajo para el estudio, análisis y elaboración de los criterios generales de acreditación, y el borrador de régimen Interior de la Comisión.

En su reunión del día 29 de julio de 1999 aprueba un acuerdo relativo a los principios generales de organización y funcionamiento del Sistema Acreditador de Actividades de Formación Continuada. Mediante dicha Resolución de la Subsecretaria de Sanidad, se establecen los requisitos mínimos que han de reunir las instituciones y organismos que solicitan reconocimiento de acreditación para poder impartir actividades de formación continuada, así como las características de las mismas para que sean válidas en su capacidad formativa.

La estructura organizativa de la Comisión de Formación Continuada del Sistema Nacional de Salud se sustenta en tres organismos: el Pleno de la Comisión de Formación Continuada, el Comité de Evaluación y la Secretaría Técnica (Tabla III).

Tabla 3. ESTRUCTURA DE LA COMISIÓN DE FORMACIÓN CONTINUADA

$\cdot$ PLENO DE LA COMISIÓN
$\cdot$ COMITÉ DE EVALUACIÓN
$\cdot$ SECRETARÍA TÉCNICA

El Pleno de la Comisión de Formación Continuada de las Profesiones Sanitarias del Sistema Nacional de Salud, constituida como Comisión Permanente del Consejo Interterritorial del Sistema Nacional de Salud, es la responsable de la Formación Continuada del Sistema Nacional de Salud, de acuerdo con las Bases del Convenio de Conferencia Sectorial. Tiene carácter de Comisión Permanente del Consejo Interterritorial. A ella corresponde el establecimiento de los criterios generales comunes y mínimos para la acreditación de actividades y de instituciones, así como la coordinación de planes y actuaciones de las comunidades autónomas.

Su composición, especificada en el Convenio de conferencia Sectorial, es la siguiente:

- Un representante de cada una de las Comunidades Autónomas.

- Un representante del Ministerio de Sanidad y Consumo.

- Un representante del Ministerio de Educación y Ciencia.

- Los Presidentes de los Consejos de Colegios Profesionales 
Con objeto de hacer operativo el Sistema Acreditador, formulado por la Comisión, existe una Secretaría Técnica dependiente de la propia Comisión. Coordinados por la Secretaría Técnica están los Comités de Evaluación que valoran técnicamente las actividades formativas que han solicitado acreditación ${ }^{4 \text { y }}$.

Cada Comité de Evaluación está integrado por expertos en formación continuada, en número no inferior a 3 ni superior a 5 . Los miembros de este Comité son designados por la Comisión de Formación Continuada de las Profesiones Sanitarias del Sistema Nacional de Salud, por períodos renovables de dos años.

Cualquier actividad a valorar por el órgano Acreditador pertinente debe estar descrita en un Formulario tipo que puede solicitarse en las Secretarías Técnicas correspondientes, y en el que se incluyen los siguientes epígrafes:

1. Datos generales sobre la actividad a acreditar y su proveedor:

- Nombre o título de la actividad y director responsable,

- Información sobre la entidad proveedora de la actividad.

- Tipo de actividad

- Número máximo de participantes.

- Duración prevista de la actividad (en horas).

- Grupo profesional al que se dirige la actividad.

- Procedimiento de financiación de la actividad.

2. Información para la valoración cualitativa:

- Objetivos de la actividad formativa. Descripción de los objetivos generales y específicos de la actividad

- Organización y logística.

- Descripción del programa detallado de la actividad, el profesorado, los recursos disponibles, el calendario, las plazas disponibles, el método de selección de los participantes y la adecuación entre la duración de la actividad y los objetivos.

- Para el caso de actividades no presenciales, es preciso señalar el tipo de soporte del material de información (material impreso, CD Rom, Internet, etc.) así como el sistema de elaboración y evaluación de manuales y cuestionarios.

- Pertinencia de la actividad, descripción de las necesidades a las que obedece la actividad y forma como han sido detectadas.

- Metodología docente. Descripción de la metodología docente que se va a utilizar y del tipo de interrelación entre los participantes, así como de éstos con el profesorado.

- Evaluación. Descripción precisa del sistema de evaluación que se va a aplicar, especificando en cada caso qué se va a evaluar: participantes, profesorado, proceso, impacto de la actividad o asistencia. También es conveniente señalar el medio o sistema empleado y los requerimientos exigidos para la concesión de créditos. El solicitante deberá especificar claramente cuáles serán los criterios de selección y/o inscripción formal de los candidatos participantes, así como los requerimientos exigidos para que el discente pueda hacerse acreedor de los créditos concedidos a la actividad.

El formulario es analizado por el Comité de Evaluación y la aprobación definitiva de la acreditación es competencia del organismo correspondiente. Si la respuesta es positiva, el proveedor del curso recibirá notificación sobre el número de créditos asignados a la actividad en cuestión, y aquellos profesionales que participen en ella tendrán derecho a beneficiarse de estos créditos.

La Comisión de Formación Continuada entiende el concepto acreditación como la valoración que un organismo externo hace, utilizando criterios y estándares previamente establecidos.

El sistema de acreditación empleado se basa en la valoración de un Componente Cualitativo (Factor Calidad), complementado con un Componente Cuantitativo (Factor Extensión) (Tabla IV).

En el Componente Cualitativo se valoran los distintos apartados que componen el perfil pedagógico de la actividad, en base a los siguientes criterios (Tabla V):

1. Objetivos de la actividad formativa. La actividad ha de quedar suficientemente explicada en sus objetivos, distinguiendo los objetivos generales y los objetivos específicos que se pretenden conseguir en el proceso de aprendizaje de los profesionales a los que va dirigida la actividad, que han de ser observa-

Tabla 4. SISTEMA ACREDITADOR

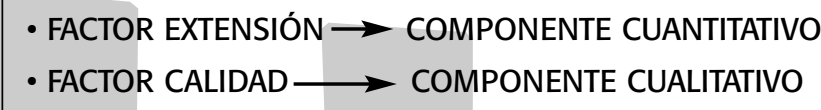

Tabla 5. PERFIL PEDAGÓGICO

- OBJETIVOS GENERALES Y ESPECÍFICOS

- ORGANIZACIÓN Y LOGÍSTICA

- PERTINENCIA DE LA ACTIVIDAD

- METOdOlOGIA DOCENTE

- EVALUACIÓN 
bles y medibles. Los primeros hacen referencia al objetivo de la acción formativa en su sentido más amplio, mientras que los segundos deben ser formulados en términos de qué área de formación se considera prioritaria (adquisición de habilidades o destrezas, mejora de actitudes del profesional, etc.). Este punto es muy importante, ya que va a condicionar la valoración de los siguientes epígrafes.

2. Organización y logística: Debe incluir la descripción lo más precisa posible del programa docente, el profesorado y su método de elección, otros recursos humanos, los recursos materiales, el calendario, los criterios de selección de aspirantes y la adecuación entre la duración de la actividad y los objetivos, profesorado y número de horas.

3. Pertinencia de la actividad. El contenido de la actividad debe responder a algún tipo de necesidad o demanda suficientemente explicitada y justificada. Es bueno indicar cómo se han detectado las necesidades formativas (encuesta, opinión de los profesionales, propuesta de una comisión, opiniones de expertos, sugerencias de la literatura especializada, etc.). En los momentos actuales, caracterizados por el trabajo en equipo, en el seno de organizaciones complejas, las propias organizaciones han de desempeñar un papel relevante cuando sea preciso formular las necesidades formativas.

4. Metodología docente. Es importante valorar en cada ocasión el grado de adecuación de la metodología a los objetivos perseguidos y a los recursos disponibles, así como el grado de interacción entre los participantes y los docentes.

5. Evaluación. Es importante que quede especificada la utilización o no de algún tipo de evaluación, ya sea de los participantes, de los docentes, de la propia actividad en cuanto a sus objetivos o del proceso formativo, etc.. En el caso de actividades presenciales, deberá especificar el sistema de control de asistencia empleado. En el caso de actividades no presenciales es necesario contar con un sistema de evaluación de conocimientos; y con objeto de asegurar al máximo la objetividad de la evaluación, se prima la realización de la misma por un agente independiente del promotor de la actividad. Mediante un proceso de feed-back permanente a partir de los resultados de la evaluación, se puede mejorar la calidad de la Formación Continuada.

Con el objeto de estandarizar la valoración de cada uno de los criterios que integran este componente cualitativo, por parte de los diversos evaluadores, se utiliza una Escala de Licker modificada, donde el valor mínimo es el 0,0 y el valor máximo 0,4.
Partiendo de la base de la importancia desigual que se concede a cada uno de los criterios se introduce un Factor de Ponderación (Tabla VI).

El componente cualitativo descrito se complementa con el Componente Cuantitativo, basado en la duración de la actividad. Este componente se modula con un factor de corrección con el objeto de introducir un elemento de equilibrio en la puntuación final de la actividad en función del número de horas (Tabla VII).

Se pretende, por tanto, primar las actividades de corta duración y penalizar las de más larga duración. El resultado de multiplicar el número de horas por el factor de corrección correspondiente, dividido todo ello entre diez, nos dará el valor de este factor de extensión ( $\mathrm{Fe}$ ) (Tabla VIII).

Los créditos totales asignados a una actividad concreta serán el resultado del producto de la puntuación del componente cualitativo por el componente cuantitativo, debidamente corregido. El resultado final es un número determinado de créditos por actividad que puede traducirse, en su caso, por créditos/hora.

En el Convenio de Conferencia Sectorial de diciembre de 1997, por el que se creaba la Comisión de Formación Continuada de las Profesiones Sanitarias del Sistema Nacional de Salud, se con-

\section{Tabla 6.}

\begin{tabular}{|ll|}
\hline Objetivos de la actividad & x 1,0 \\
Organización y Logística & x 1,0 \\
Pertinencia de la actividad & $\times 2,0$ \\
Metodología docente & $\times 1,5$ \\
Evaluación & $\times 1,5$ \\
\hline
\end{tabular}

Tabla 7.

\begin{tabular}{|ll|}
\hline$<20$ horas & x 1,1 \\
Entre 21 y 40 horas & x 1,0 \\
Entre 41 y 80 horas & x 0,9 \\
$>$ 80horas & x 0,7 \\
\hline
\end{tabular}

Tabla 8.

$$
\mathrm{Fe}=\frac{\text { Duración (en horas) } x \text { Factor de Corrección }}{10}
$$


templaba explícitamente el papel de las comunidades autónomas en el ámbito de la formación continuada y su acreditación.

En todos estos casos, el funcionamiento es idéntico al de la comisión Central. Desde el punto de vista estructural, reproducen más o menos la composición de la Comisión Central y todas ellas cuentan con una Secretaría Técnica y una comisión de AcreditaciónEvaluación.

El formulario de solicitud de acreditación utilizado es común para todas, como comunes son asimismo los criterios de evaluación de las actividades sometidas a acreditación por los distintos proveedores. En consecuencia, los créditos son válidos en todo el territorio nacional.

Este desarrollo territorial en sus diez años de funcionamiento ha demostrado su factibilidad $\mathrm{y}$ su impacto sobre la oferta formativa. Las previsiones de su desplegamiento autonómico se están cumpliendo y sólo faltan las Comunidades Autonomas de Canarias, Cantabria, Castilla La Mancha, Murcia y Asturias para ser operativo en su totalidad.

En la actualidad, desde el año 2003, los profesionales sanitarios contamos con tres importantes normativas que contemplan diversos aspectos relacionados con la formación continuada y con su sistema acreditador. Son la Ley de Cohesión y Calidad, el Estatuto Marco, y la Ley de Ordenación de las Profesiones Sanitarias (Tabla IX).

La Ley 16/2003, de 28 de mayo, de Cohesión y Calidad del Sistema Nacional de Salud ${ }^{6}$, establece acciones de coordinación y cooperación de las Administraciones Públicas Sanitarias como medio para asegurar a los ciudadanos el derecho a la protección de la salud, con el objetivo común de garantizar la equidad, la calidad y la participación social en el Sistema Nacional de Salud.

\section{Figura 9. REGULACIÓN NORMATIVA ACTUAL DE LA FORMACIÓN CONTINUADA}

- LEY DE COHESIÓN Y CALIDAD

- eStatuto maRco

- LEY DE ORDENACIÓN DE LAS PROFESIONES SANITARIAS

\section{Tabla 10.}

ESTABLECIMIENTO DE CRITERIOS COMUNES

PARA ORDENAR LA FORMACIÓN CONTINUADA

(LEY DE COHESIÓN Y CALIDAD)
La Ley se estructura en un capítulo preliminar y otros once capítulos. El capítulo III contiene básicamente principios referidos a la planificación y formación de los profesionales de la sanidad. Principal interés tiene la creación de una Comisión de Recursos Humanos que tiene encomendadas, entre otras, las competencias en materia de formación continuada.

En su artículo 38 señala que son las Administraciones Públicas las encargadas de establecer criterios comunes para ordenar las actividades de formación continuada. Así mismo, indica que el Ministerio de Sanidad y Consumo y los órganos competentes de las Comunidades Autónomas podrán delegar las funciones de gestión y acreditación de la formación continuada en corporaciones o instituciones de derecho público (Tabla X).

En relación con el desarrollo profesional, la Ley determina que deberá responder a criterios comunes en relación con los ámbitos de la formación continuada, la carrera profesional y la evaluación de competencias.

La Ley 55/2003, de 16 de diciembre, del Estatuto Marco del personal estatutario de los servicios de salud (7), deroga el régimen estatutario preconstitucional sustituyéndolo por el marco básico que compone el propio estatuto y por las disposiciones que, en el ámbito de cada Administración Pública, desarrollen tal marco básico y general.

El contenido de la Ley se estructura en 14 capítulos, a través de los cuales se regulan los aspectos generales y básicos de las diferentes materias que componen el régimen jurídico del personal estatutario. En el apartado correspondiente a derechos y deberes del personal estatutario de los servicios de salud destaca el derecho a la formación continuada adecuada a la función desempeñada y al reconocimiento de su cualificación profesional en relación a dichas funciones. Como deber, establece el mandato de mantener debidamente actualizados los conocimientos y aptitudes necesarios para el correcto ejercicio de la profesión (Tabla XI).

La Ley 44/2003, de 21 de noviembre, de Ordenación de las Profesiones Sanitarias (LOPS) ${ }^{8}$, tiene por finalidad dotar al sistema sanitario de un marco legal que contemple los diferentes instrumentos y recursos que hagan posible la mayor integración de los profesiona-

\section{Tabla 11.}

LA FORMACIÓN CONTINUADA COMO DERECHO Y DEBER DEL PROFESIONAL SANITARIO (ESTATUTO MARCO) 
les en el sistema sanitario, tanto en la vertiente pública como en la privada.

El ejercicio profesional, público o privado, se regula por esta Ley. Entre sus principios rectores se encuentra la necesidad de que los profesionales sanitarios realicen a lo largo de su vida profesional una formación continuada. Para ello se deberá poder disponer de toda la estructura del sistema sanitario para ser utilizada en la formación continuada del profesional (Tabla XII).

\section{Tabla 12.}

\section{NECESIDAD DE QUE LOS PROFESIONALES SANITARIOS \\ REALICEN, A LO LARGO DE SU VIDA PROFESIONAL, UNA FORMACIÓN CONTINUADA}

Define la formación continuada como un proceso de enseñanza y aprendizaje activo y permanente al que tienen derecho y obligación los profesionales sanitarios, que se inicia al finalizar los estudios de pregrado o de especialización y que está destinado a actualizar y mejorar los conocimientos, habilidades y actitudes de los profesionales sanitarios ante la evolución científica y tecnológica y las demandas y necesidades, tanto sociales como del propio sistema sanitario.

Entre las funciones que la LOPS atribuye a la Comisión de Formación Continuada, cabe destacar la de proponer actividades y actuaciones de formación continuada de carácter prioritario y común para el conjunto del sistema sanitario. Así mismo procederá al establecimiento de criterios y requisitos para la acreditación de actividades de formación continuada.

$\mathrm{Y}$ establece que la acreditación, realizada de acuerdo con los requisitos, procedimientos y criterios establecidos, tendrá efecto en todo el territorio nacio- nal, sea cual sea la Administración Pública que expidió la acreditación. Asimismo, sólo podrán ser subvencionadas, con cargo a fondos públicos, las actividades de formación continuada acreditadas.

En conclusión, es necesario que tengamos presente el valor estratégico de los sistemas de formación continuada como elementos orientados a la motivación e incrementación de los conocimientos del médico, así como su importancia en su desarrollo profesional continuo.

\section{BIBLIOGRAFIA}

1. Harvard Business Review on Knowledge Management. Boston: Harvard Business School Press; 1998.

2. Pardell, H. "Qué es y para qué sirve la Formación Médica Continuada. En: Pardell H, director. Formación Médica Continuada, a debate”. Barcelona: Ediciones Mayo. 2001. 7-11

3. Convenio de Competencia Sectorial del Consejo Interterritorial del Sistema Nacional de Salud sobre Formación Continuada de las Profesiones Sanitarias. Resolución de 22 de diciembre de 1997, de la Subsecretaría. BOE de 13 de febrero de 1998.

4. Pallarés L. El sistema de acreditación de la Comisión de Formación Continuada del Sistema Nacional de Salud y de las comisiones Autonómicas. En: Pardell H, director Industria farmacéutica y formación médica continuada. Barcelona: Ediciones MAYO; 2003. 47-61.

5. A. Gual, L. Pallarés. Cómo se acreditan las actividades de formación médica continuada. En Pardell H., Director. Formación médica continuada, a debate. Ediciones Mayo. 2001. 17-21.

6. Ley $16 / 2003$, de 28 de mayo, de cohesión y calidad del Sistema Nacional de Salud. BOE ${ }^{\circ} 128$.

7. Ley 55/2003, de 16 de diciembre, del Estatuto Marco del personal sanitario de los servicios de salud. BOE n ${ }^{\circ} 301$

8. Ley $44 / 2003$, de 21 de noviembre, de ordenación de las profesiones sanitarias. BOE $\mathrm{n}^{\circ} 280$, de 22 de noviembre de 2003. 41442-41458. 